doi: http://dx.doi.org/10.7124/bc.0009ED

\section{L-4. Cell model of inducible AML1-ETO translocation}

\author{
Nikolai A. Lomov, Vladimir S. Viushkov, \\ Mikhail A. Rubtsov \\ M.V. Lomonosov Moscow State University, Mos- \\ cow, Russia LIA LFR20 (LIA French-Russian Can- \\ cer Research Laboratory) Villejuif, France - Moscow, \\ Russia I.M. Sechenov First Moscow State Medical \\ University (Sechenov University), Moscow, Russia \\ lomov13@gmail.com
}

Recent progress in cancer therapy allows patients to live longer. However, the increase in life expectancy raises the chance that the longterm side effects of therapy will manifest. Among these is secondary (so-called treatment-related) leukemia (tAML). This disease arises as a consequence of anti-cancer therapy with DNA topoisomerase II inhibitors (i.g. etoposide). It was shown that specific chromosomal translocations between AML1 (RUNX1) and ETO (RUNX1T1) genes can be found in many cases of tAML [1]. A widely accepted hypothesis postulates that this translocation is a consequence of the erroneous repair of DNA double-strand breaks induced by drug-poisoned DNA topoisomerase II [2]. One possible way to induce AML1-ETO translocation is to treat cells with etoposide. However, the occurrence of this particular translocation, in this case, will be extremely low. Therefore, we aimed to create a cell model with inducible targeted chromosomal breaks in AML1 and ETO genes to study the formation of leukemogenic chromosomal translocation. Methods: An integrative construction with inducible Cas9 gene and genes for guide RNAs to AML1 and ETO genes was obtained. The expression of Cas9 gene was under the control of TetONsystem. Guide RNAs were tested by ENITapproach [3]. This construction was integrated into the genome of LCL (RPMI 8866) cells by means of homologous recombination into AAVS1 locus. The induction of Cas9 expression by doxycycline was assessed by qRTPCR. The induction of translocation was verified by PCR, and the frequency of translocation was quantified by qPCR. Results: We create the transgenic cells with the Cas9 expressed upon activation with doxycycline. The expression reaches a plateau at 24 hours post activation. Cas 9 introduces breaks into two specific loci. As a result, the desired AML1ETO translocation is formed. Conclusions: The obtained cell model with inducible AML1ETO translocation can be used to study early events and to investigate details of molecular mechanisms leading to AML1-ETO-associated secondary leukemia. In addition, this model is suitable for screening for new drugs, preventing such unfavorable side-effects of chemotherapy.

Work was partially supported by RFBR grants \# 19-0400531_A and 19-54-16002_CNRS_a.

References: Rowley J.D., Olney H.J., 2002. International Workshop on the relationship of prior therapy to balanced chromosome aberrations in therapy-related myelodysplastic syndromes and acute leukemia: Overview report // Genes Chromosom. Cancer. V. 33. № 4. P. 331345. Cowell I.G., Austin C.A., 2012. Mechanism of generation of therapy related leukemia in response to antitopoisomerase II agents // Int. J. Environ. Res. Public Health. V. 9. № 6. P. 2075-2091. Germini D., Bou Saada Y., Tsfasman T., Osina K., Robin C., Lomov N., Rubtsov M., Sjakste N., Lipinski M., Vassetzky Y., 2017. A OneStep PCR-Based Assay to Evaluate the Efficiency and Precision of Genomic DNA-Editing Tools // Mol. Ther. Methods Clin. Dev. V. 5. № June. P. 43-50. 